\title{
1 The voltage-sensing mechanism of the KvAP
}

\section{2 channel involves breaking of the S4 helix}

3 Olivier Bignucolo1,2,3,\#, Simon Bernèche1,2

4 1SIB Swiss Institute of Bioinformatics 2Biozentrum, University of Basel, Switzerland

5 3Current address: Faculty of Biology and Medicine, University of Lausanne, Switzerland

6 \#Correspondence: olivier.bignucolo@unil.ch

\section{Abstract}

8 Voltage-gated ion channels allow ion permeation upon changes of the membrane

9 electrostatic potential $(\mathrm{Vm})$. Each subunit of these tetrameric channels is composed of six transmembrane helices, of which the anti-parallel helix bundle S1-S4 constitutes the voltage-

11 sensor domain (VSD) and S5-S6 forms the pore domain. Here, using molecular dynamics (MD) simulations, we report novel responses of the archaebacterial potassium channel KvAP to cell polarization. We show that the S4 helix, which is straight in the experimental crystal structure solved under depolarized conditions (Vm 0), breaks into two segments when the cell is polarized $(\mathrm{Vm} \ll 0)$, and reversibly forms a single straight helix following depolarization of the cell $(\mathrm{Vm}=0)$. The outermost segment of S4 translates along the normal to the membrane, bringing new perspective to previously paradoxical accessibility experiments that were initially thought to imply the displacement of the whole VSD across the membrane. Our simulations of KvAP reveal that the breaking of S4 under polarization is not a feature unique to hyperpolarization activated channel, as might be suggested by recent cryo-EM structures and MD simulations of the HCN channel. 


\section{Introduction}

Voltage-gated potassium channels (Kv) are tetramers that open and close as a function of the membrane electrostatic potential (1). Each subunit is composed of six transmembrane helices S1-S6. Voltage dependence is granted by helices S1 to S4, an anti-parallel helical bundle constituting the voltage-sensor domain (VSD), which is linked to the pore domain composed of helices S5 and S6. A much-conserved structural feature of the voltage-sensor domains is a series of four to six basic residues distributed along the S4 helix, each one followed by two hydrophobic residues. The voltage-sensing properties are attributed to these positively charged residues, which are assumed to respond to the membrane electrostatic potential $(\mathrm{Vm})$ by a translation along the membrane normal. This results in an apparent charge transport, or gating current (2-4). Under depolarized potential, the pore is open and the channel enters its active state, which can be determined experimentally. The resting or closed state under polarized potentials has been more challenging to investigate.

A "consensus" mechanism describing the voltage-dependent conformational changes of $\mathrm{Kv}$ channels in response to variations of the membrane electrostatic potential was developed (5), through the integration of several computational studies, notably based on the structures of the Kv1.2 and Kv1.2/2.1 chimera channels (6-15). The proposed model consists, within a few Angstroms uncertainty, of a sliding helix mechanism in which S4 undergoes an ensemble of transitions towards a resting state, involving a rotation and a translation along its main axis and toward the intracellular compartment. The translation is $\sim 10 \AA$ long, with a spread of 3-4A. While most of the current knowledge on eukaryotic Kv channels was incorporated in this model, data from the archaea KvAP channel seemed incompatible with the proposed mechanism.

The structure of KvAP voltage-sensing domain was solved by crystallography and NMR (1618), and more recently its complete structure was solved by cryo-EM (19). These 
experiments being performed in absence of any membrane voltage, only the active state of the KvAP VSD could be captured. Its elusive resting state has nevertheless been characterized by several biophysical studies (20-23). The sliding helix model depends on the possibility of S4 to exert a translation along its axis in response to variation of the membrane electrostatic potential. As shown in Figure 1A-C, while such a movement is plausible for Kv1.2 and Kv1.2/Kv2.1, there is essentially no room for the long S4 helix of KvAP (33 vs 20 residues) to slide upon depolarization without exposing hydrophobic residues to the polar environment of phospholipid head groups or the solvent.

One key experimental observation, for which a mechanistic explanation remains elusive since its publication, is the accessibility measurements of avidin to biotinylated cysteins in the S3 and S4 segments $(21,23)$. These experiments showed that avidin in the intracellular space could bind to biotinylated cysteine located in the middle of S4. This observation was explained by a large displacement of the whole S4 helix across the hydrophobic core of the membrane, which was difficult to reconcile with other experiments (24).

Our unrestrained simulations show that, under cell polarization, the voltage-sensing domain of KvAP undergoes a transition that involves the rupture of the Asp62-Arg133 salt bridge immediately followed by the formation of a kink in the middle of S4. The resulting sliding movement of the kinked S4 helix towards the intracellular space provides a more consensual explanation to the avidin accessibility experiments. Similar conformational changes were recently observed in the VSD of HCN, a channel activated by hyperpolarization $(25,26)$. The breaking of S4 observed in the VSD of KvAP reveals that this transition is not specific to hyperpolarization gating. Sequence alignment reveals that the specific sequence of the KvAP S4 N-ter is hardly found in any eukaryotic voltage-gated ion channel (see Table 1). However, this sequence is found in several archaea and prokaryotes, among which many pathogens, making it a potential selective target for antibiotic investigations. 


\section{KvAP response to cell polarization involves bending of S4}

To address the ill-defined mechanism of voltage-sensing in prokaryotic cells, we carried out a large number of independent molecular dynamics (MD) simulations in which the VSDs were exposed to a wide range of membrane electrostatic potentials. As shown in Figure 2, a system consisted of two bilayers mimics a cell with two separated water compartments that, according to the orientation of the bilayer leaflets and incorporated proteins, correspond to the extra- and intracellular compartments. This compartmentalization allows one to adjust the membrane potential by changing the number of ions in either compartment (27-29). We constructed 66 such systems, allowing the study of 132 VSDs. The simulated membrane potential $(\mathrm{Vm})$ ranged from -1.7 to $0.5 \mathrm{~V}$. While these $\mathrm{Vm}$ values are of higher magnitude than physiologically found in cells, they remain in a range that does not expose the membrane to electroporation.

We controlled the electrostatic steadiness of the bilayer systems by monitoring the difference $\Delta \mathrm{Vm}$ between the membrane potential averaged over the first and last $20 \mathrm{~ns}$ of the trajectory (see Figure 2). As a consequence of the limited size of the systems, which typically contain $\sim 240000$ atoms, a single charge transport across the membrane induces a $\Delta \mathrm{Vm}$ of $\sim 200 \mathrm{mV}$. In four of the 66 simulations we detected variation of membrane potential that indicated charge transport equivalent to the relocation of one or two ions across the membrane. As shown in Table 1, these events occurred only when the membrane was polarized (negative potential) (Fisher's exact test probability $\mathrm{p}=0.007$ ).

The crystal structure of KvAP is characterized by two funnels readily accessible to the solvent, as can be seen in Figure 1C. An important salt bridge (figure 3) between Asp62 (helix 
S1) and Arg133 (helix S4) in the middle of the membrane constitutes the only barrier between the extra- and intracellular compartments. We observed the rupture of this salt bridge in all simulations in which a charge transport occurred (Table 1, Figures 3 and 4), and in none of the others. Upon rupture of the salt bridge, the negative charge of Asp62 moved toward the extracellular compartment, while the positive charge of Arg133 moved toward the intracellular compartment, which resulted in the observed gating charge transport. In addition, whereas the S4 helix is straight under depolarized conditions, it formed the evoked kink at the level of Gly134 only when the cell was polarized and the salt bridge was broken. Consequently, S4 was split in two segments, the one on the intracellular side being reoriented in a direction almost parallel to the membrane surface (Figure 3, right), like the S4-S5 linker of Kv1.2 and Kv1.2/Kv2.1 (Figures 1A and 1B).

A time series analysis of the trajectories in which the gating charge transport occurred shows that the breaking of the salt bridge and the kink in S4 occur concurrently (Figure 4A). None of these events was observed in the simulations conducted at Vm $\sim 0 \mathrm{~V}$ (Figure 4A, inset). This time series analysis supports the ideas that 1) the two conformational changes are related and 2) they are due to the membrane polarization. We further asked whether the membrane potential alone drives the status of the Asp62-Arg133 salt bridge. In a particular simulation initialized under a strong polarizing potential, the salt bridge broke after $\sim 90 \mathrm{~ns}$. Consecutively to the reorientation of the Asp62 and Arg133 side chains, the membrane potential decreased within $\sim 30$ ns to a value corresponding to a gating charge transport of two units and remained stable during the next 50-60 ns. With the aim of testing the dependence of the conformational changes on the membrane potential, we stopped the simulation and moved ions between the extra- and intracellular compartment in order to mimic a depolarized potential. We then continued the simulation from this new starting 
124 point. Within $\sim 10$ ns of simulation, the side chains of Asp62 and Arg133 reoriented and restored the salt bridge, which remained intact for the next 200 ns (Figure 4B).

Interestingly, experimental $(17,18,20,30)$ and computational $(31)$ investigations have reported either that the S4 of KvAP may kink near the middle of the bilayer, or that the Asp62Arg133 salt bridge may break (32). In an NMR structure determination of the KvAP VSD, a loss of helical periodicity was identified at the level of Gly134, suggesting that the S4 helix might

131 be constituted of two helices connected by a hinge comprising Ile131, Ser132 and Arg133 (18).

132 Interestingly, three of the 20 conformations deposited for the KvAP VSD NMR solution 133 structure (code $2 \mathrm{KYH}$ ) display a kink in the middle of S4 (17).

134 Whereas experimental or computational studies support the idea of a hinge in the middle of 135 S4 or the rupture of the salt bridge, the present study shows for the first time that these two 136 conformational changes happen simultaneously upon polarization and that they lead to the 137 charge transport observed experimentally and generally interpreted as a gating current.

\section{Avidin binding to biotinylated KvAP voltage-sensor domain}

140 In 2003 and 2005, Jiang et al.(23) and Ruta et al.(21) reported experiments in which the 141 binding of avidin to biotinylated cysteines was used to deduce the residue accessibility from 142 the external or internal cell compartments. In the study described by Jiang et al., a $17 \AA ̊$ linker 143 connected the Cys $\mathrm{C} \alpha$ atom to biotin through an amide bond. These avidin binding 144 experiments notably showed that the biotinylated residues 125 and 127 , located in the 145 upper half of the S4 helix, were accessible to avidin from the intracellular side. These results 146 supported the idea of a voltage-sensor paddle model in which the helix-turn-helix S3a-S4 147 moves through the membrane upon voltage changes. However, this model requires an 148 important movement of charged amino acids across the hydrophobic core of the membrane, 149 which was difficult to reconcile with other observations(24). 
151 Our MD simulations show that the bending of S4 induced by membrane polarization reduces 152 sufficiently the distance required for avidin to bind to the biotinylated residues without 153 requiring the $\mathrm{S} 3 \mathrm{a}$ and $\mathrm{S} 4$ helices to cross the membrane core. In order to determine how 154 close an avidin molecule could come to the residues investigated by Jiang et al. (23) and Ruta et al. (21) considering the conformational change of S4 under membrane polarization, we conducted a steered molecular dynamics (SMD) investigation involving the KvAP VSD and the monomeric avidin-biotin complex (Figure 5A). A soft constant pulling force (see Methods) was exerted between the biotin carboxylic acid functional group and the C $\alpha$ of Ile127. We performed the steered molecular dynamics simulations (SMD) starting either from 160 the X-ray structure or from a conformation displaying the kink in S4 described above, with 161 an initial distance between the $\mathrm{C} \alpha$ of Ile127 and the biotin carboxylic acid of $\sim 25-26 \AA$. 162 Whereas this distance stabilized to $\sim 19 \AA$ during 50 ns of constant pulling in the case of a 163 VSD with a straight S4 helix, it decreased steadily, attaining $\sim 11 \AA$, and stabilized to a value 164 of $\sim 13 \AA$ in the case of the kinked S4 helix (Figure 5). This distance is significantly less than 165 the experimentally used linker, and thus the response of the VSD to membrane polarization 166 is compatible with the accessibility experiments described above.

\section{Discussion}

169 This study reveals a novel response of KvAP to cell polarization that consists in the 170 concurrent disruption of the salt bridge between Asp62 in S1 and Arg133 in S4 and the 171 formation of a kink at the level of Gly134 in S4. Whereas the rupture of the Asp62-Arg133 salt 172 bridge has been reported as a response to polarization (33), the current study shows that 173 the rupture of the salt bridge induces the formation of a kink in S4. The induced conformation 
174 is expected to further allow the translation of the upper part of S4 along its principal axis and

175 towards the intracellular side of the bilayer.

176 The helix breaking occurs at the level of a Gly residue located 8 positions downstream from

177 the S4 basic residues. Sequence alignments reveal that the specific features of the KvAP S4

178 sequence are not seen in any eukaryotic voltage-gated ion channel, whereas there are found

179 in several archaea and prokaryotes, among which many pathogens, as further explained in

180 Supplementary Material.

181 The observed conformational changes imply that a tethered biotin on the external half of S4

182 is accessible to avidin from the intracellular compartment, bringing a biophysically coherent

183 explanation to the accessibility experiments described by Jiang et al (23), and Ruta et al (21).

184 Studies involving both the voltage-sensor and the pore would be required to further

185 investigate the postulated function of the lower half of S4 as a surrogate S4-S5 linker, notably

186 in the non-domain-swapped architecture revealed by a recent cryo-EM structure of KvAP 187 (19).

In a recent cryo-EM study on the HCN channel, a hyperpolarization activated potassium 190 channel, a disulfide bridge was generated between F186C in helix S2 and S264C in helix S4 191 with the aim of mimicking hyperpolarized conditions. Consequently, the VSD was trapped in 192 a presumably activated state, characterized by a kink in S4 at the level of the disulfide bridge and a sliding movement of the external part of S4 towards the intracellular side (25). Similar

194 conformational changes were observed in MD simulations of the HCN voltage-sensor domain 195 under membrane hyperpolarization (26). The breaking of the S4 helix in two smaller helices 196 was suggested to be essential to hyperpolarization gating. Our unrestrained simulations 197 reveal that this feature is not unique to channel activated by cell hyper polarization, but is 198 also observed in a channel activated by depolarization, like KvAP. 


\section{Methods}

Sequence analysis. The non-redundant UniProt/SwissProt sequence database was used for searching voltage-gated potassium channels homologs to the KvAP VSD sequence, ID Q9YDF8 $(34,35)$. The sequences were further curated using in-house Python scripts in order to remove undefined species, uncharacterized fragments, retain sequences of length similar to the KvAP VSD \pm 100 residues. The scripts further selected sequences characterized by the typical feature of a voltage-sensor domain, i.e. a series of three triplets consisting of a pair of mostly hydrophobic residues followed by a basic residue and, in addition, a segment of seven any residues followed by a Gly. This last criterion allowed us to discriminate the sequences according to the specificity of the S4 helix described in this work.

MD simulations. The atomic model of the KvAP VSD was based on the crystal structure PDB code 10RS, assumed to correspond to the active state of the channel (36). The structure of a complete KvAP channel was solved recently through cryo-EM (19). Note, however, that the structure of the voltage-sensor domain is identical in the previous and new structures (see suppl. Figure 2). Specifically, S4 is straight in both structures, which were solved at Vm $=0 \mathrm{~V}$. The VSD was inserted in an asymmetric bilayer using the CHARMM-GUI web service (37). The "extra-cellular" leaflet was composed of 100 POPC (1-palmitoyl-2-oleoyl-sn-glycero-3-

217 phosphocholine) and 80 cholesterol, and the "intra-cellular" leaflet was composed of 50

218 POPC, 50 POPS (1-palmitoyl-2-oleoyl-sn-glycero-3-phosphoserine) and 80 cholesterol 219 molecules. The system was further solvated with $\sim 25,000$ water molecules, represented by 220 the TIP3P model (38). Neutralizing $\mathrm{K}+$ and $\mathrm{Cl}$ - counterions were added to mimic a salt 221 concentration of $0.15 \mathrm{M}$. Two such systems were combined in an antiparallel way to form a 222 double bilayer system, simulating a cell membrane separating two different water compartments $(28,39,40)$. The system contained $\sim 235,000$ atoms. The construct contains 
in its center a water slab simulating the "intracellular" compartment, and the two slabs on the edges are combined through periodic boundary conditions to form the "extracellular" compartment. Using this construct, the membrane potential can be adjusted to the desired value by changing the number of ions in either compartment.

The simulated membrane potential $(\mathrm{Vm})$ ranged from -1.7 to $0.5 \mathrm{~V}$. These high values allowed to augment the conformational space exploration, however without attaining potentials that would induce electroporation. Thus, in a study investigating the stabilization effect of cholesterol on lipid membranes, Casciola et al. (41) exposed bilayers composed of 0 to $50 \mathrm{~mol} \%$ cholesterol to membrane potentials values up to $5.35 \mathrm{~V}$. They observed within the first 60-70 ns of simulation that the "electroporation thresholds increased from $\sim 2.3 \mathrm{~V}$ for bare bilayers to $\sim 4.4 \mathrm{~V}$ as the cholesterol content reached $30 \mathrm{~mol} \%$ concentration". In another study, an electroporation thresholds of $-1.8 \mathrm{~V}$ was reported for a cholesterol free membrane(42). According to these data, the membrane potential applied in our work is not expected to destabilize the bilayers, which contain $\sim 45$ mol\% cholesterol. We effectively 238 did not observe any strong membrane deformation during the simulations, generally of 239200 ns length, reaching 740 ns in one case.

240 All-atom MD simulations were performed with the GROMACS software package version 4.5(43), with the CHARMM force-field (44), versions v27 for proteins(45) and v36 for 242 lipids(46). A constant pressure of 1 bar was maintained using the Berendsen algorithm (time constant 1ps) (47). The temperature was kept at $310 \mathrm{~K}$ by a stochastic rescaling of the 244 velocities (time constant $0.2 \mathrm{ps}$ ) (48). Bond lengths and angles involving hydrogen atoms 245 were constrained using the LINCS algorithm(49), allowing an integration time step of 2 fs. 246 Short-range electrostatics were cut off at $1.2 \mathrm{~nm}$, and the particle mesh Ewald method was used for long-range electrostatic (50). Van der Waals interactions were described with 248 Lennard-Jones potential up to a distance of $1.2 \mathrm{~nm}$. The systems were equilibrated following 
the CHARMM-GUI protocol(51). Independent simulations were conducted on 66 double bilayer systems, thus allowing the study of 132 voltage-sensor domains at various membrane potentials.

252 For the study of the avidin accessibility, we reasoned that whereas avidin generally forms a 253 tetramer in solution, with extremely high affinity to biotin ( $\left.\mathrm{K}_{\mathrm{d}} \sim 10-15 \mathrm{M}\right)$, it was shown that 254 the monomeric avidin also binds biotin with high affinity $\left(\mathrm{K}_{\mathrm{d}} \sim 10_{-7 \mathrm{M}}\right)$, which is sufficient to explain the experiments mentioned in the main text(52). The access of a monomeric avidin to a VSD-bound biotin is structurally less constrained than that of a tetramer. Consequently, chain A from the complex avidin-biotin crystal structure (PDB code 1AVD) was placed manually at a few angstroms from a bilayer containing KvAP, which was in either the crystallized conformation or after formation of the kink in S4 (Figure 5B shows the initial 260 positions of the molecules in this case). Force field parameters for biotin were generated 261 using the CGenFF web-service (53) based on a structure obtained from the Zinc12 database 262 (54). Constant pulling forces of $100 \mathrm{~kJ} \bullet \mathrm{mol}-1 \bullet \mathrm{nm}-1$ were applied between the C $\alpha$ atom of Ile127 263 of KvAP and the center of mass of the biotin carboxylic acid group. An equal force was applied 264 between the center of mass of the nitrogen and sulphur atoms of biotin and that of Trp70 and 265 Trp97 of avidin, as these two residues define the biotin-avidin binding site(55).

\section{Author Contributions}

267 O.B. and S.B conceived the study. O.B. performed the numerical simulations and the 268 analytic calculations. O.B and S.B wrote the manuscript.

\section{Competing financial interests}

270 The authors declare no competing financial interests. 


\section{Acknowledgments}

272 This work was supported by a grant from the Swiss National Science Foundation (SNF

273 Professorship No PP00P3_139205 to S.B.). Simulations were performed at the Swiss National

274 Supercomputing Centre (CSCS) under projects ID S545 and SM09, and data analysis at

275 sciCORE, the scientific computing center from the University of Basel

276 (http://scicore.unibas.ch/). The authors thank Annaïse Jauch and Niklaus Johner for helpful

277 comments on the manuscript.

\section{References}

$280 \quad$ 1. K. J. Swartz, Sensing voltage across lipid membranes. Nature 456, 891-897 (2008).

281 2. Q. Li, S. Wanderling, P. Sompornpisut, E. Perozo, Structural basis of lipid-driven conformational transitions in the KvAP voltage-sensing domain. Nat Struct Mol Biol 21, 160-166 (2014).

3. B. L. Tempel, D. M. Papazian, T. L. Schwarz, Y. N. Jan, L. Y. Jan, Sequence of a probable potassium channel component encoded at Shaker locus of Drosophila. Science 237, 770-775 (1987).

4. F. Bezanilla, How membrane proteins sense voltage. Nat Rev Mol Cell Biol 9, 323-332 (2008).

5. E. Vargas et al., An emerging consensus on voltage-dependent gating from computational modeling and molecular dynamics simulations. J Gen Physiol 140, 587594 (2012).

6. W. Treptow, M. Tarek, M. L. Klein, Initial response of the potassium channel voltage sensor to a transmembrane potential. J Am Chem Soc 131, 2107-2109 (2009).

7. M. Nishizawa, K. Nishizawa, Molecular dynamics simulation of Kv channel voltage sensor helix in a lipid membrane with applied electric field. Biophys $J$ 95, 1729-1744 (2008).

8. V. Yarov-Yarovoy, J. Schonbrun, D. Baker, Multipass membrane protein structure prediction using Rosetta. Proteins 62, 1010-1025 (2006).

9. L. Delemotte, W. Treptow, M. L. Klein, M. Tarek, Effect of sensor domain mutations on the properties of voltage-gated ion channels: molecular dynamics studies of the potassium channel Kv1.2. Biophys J 99, L72-74 (2010).

10. Christine S. Schwaiger, P. Bjelkmar, B. Hess, E. Lindahl, 310-Helix Conformation Facilitates the Transition of a Voltage Sensor S4 Segment toward the Down State. Biophysical Journal 100, 1446-1454 (2011).

11. E. Vargas, F. Bezanilla, B. Roux, In search of a consensus model of the resting state of a voltage-sensing domain. Neuron 72, 713-720 (2011).

12. E. J. Denning, P. S. Crozier, J. N. Sachs, T. B. Woolf, From the gating charge response to pore domain movement: initial motions of Kv1.2 dynamics under physiological voltage changes. Mol Membr Biol 26, 397-421 (2009). 
13. M. Nishizawa, K. Nishizawa, Coupling of S4 helix translocation and S6 gating analyzed by molecular-dynamics simulations of mutated Kv channels. Biophys $J$ 97, 90-100 (2009).

14. C. Domene, Voltage-sensor cycle fully described. Proc Natl Acad Sci U S A 109, 83628363 (2012).

15. L. Delemotte, M. Tarek, M. L. Klein, C. Amaral, W. Treptow, Intermediate states of the Kv1.2 voltage sensor from atomistic molecular dynamics simulations. Proc Natl Acad Sci U S A 108, 6109-6114 (2011).

16. Y. Jiang et al., X-ray structure of a voltage-dependent $\mathrm{K}+$ channel. Nature 423, 33-41 (2003).

17. J. A. Butterwick, R. MacKinnon, Solution structure and phospholipid interactions of the isolated voltage-sensor domain from KvAP. J Mol Biol 403, 591-606 (2010).

18. Z. O. Shenkarev et al., NMR structural and dynamical investigation of the isolated voltage-sensing domain of the potassium channel KvAP: implications for voltage gating. $J$ Am Chem Soc 132, 5630-5637 (2010).

19. X. Tao, R. MacKinnon, Cryo-EM structure of the KvAP channel reveals a non-domainswapped voltage sensor topology. Elife 8, (2019).

20. L. G. Cuello, D. M. Cortes, E. Perozo, Molecular architecture of the KvAP voltagedependent K+ channel in a lipid bilayer. Science 306, 491-495 (2004).

21. V. Ruta, J. Chen, R. MacKinnon, Calibrated measurement of gating-charge arginine displacement in the KvAP voltage-dependent K+ channel. Cell 123, 463-475 (2005).

22. H. Biverstahl, J. Lind, A. Bodor, L. Maler, Biophysical studies of the membrane location of the voltage-gated sensors in the HsapBK and KvAP K(+) channels. Biochim Biophys Acta 1788, 1976-1986 (2009).

23. Y. Jiang, V. Ruta, J. Chen, A. Lee, R. MacKinnon, The principle of gating charge movement in a voltage-dependent K+ channel. Nature 423, $42-48$ (2003).

24. C. Ahern, Stirring up controversy with a voltage sensor paddle. Trends in Neurosciences 27, 303-307 (2004).

25. C. H. Lee, R. MacKinnon, Voltage Sensor Movements during Hyperpolarization in the HCN Channel. Cell, (2019).

26. M. A. Kasimova et al., Helix breaking transition in the $\mathrm{S} 4$ of $\mathrm{HCN}$ channel is critical for hyperpolarization-dependent gating. Elife 8, (2019).

27. A. A. Gurtovenko, Asymmetry of lipid bilayers induced by monovalent salt: atomistic molecular-dynamics study. J Chem Phys 122, 244902 (2005).

28. A. A. Gurtovenko, I. Vattulainen, Calculation of the electrostatic potential of lipid bilayers from molecular dynamics simulations: methodological issues. J Chem Phys 130, 215107 (2009).

29. E. J. Denning, T. B. Woolf, Double bilayers and transmembrane gradients: a molecular dynamics study of a highly charged peptide. Biophys J 95, 3161-3173 (2008).

30. S. Chakrapani, L. G. Cuello, D. M. Cortes, E. Perozo, Structural dynamics of an isolated voltage-sensor domain in a lipid bilayer. Structure 16, 398-409 (2008).

31. Z. A. Sands, A. Grottesi, M. S. Sansom, The intrinsic flexibility of the Kv voltage sensor and its implications for channel gating. Biophys J 90, 1598-1606 (2006).

32. J. A. Freites, D. J. Tobias, Voltage Sensing in Membranes: From Macroscopic Currents to Molecular Motions. J Membr Biol 248, 419-430 (2015).

33. J. A. Freites, E. V. Schow, S. H. White, D. J. Tobias, Microscopic origin of gating current fluctuations in a potassium channel voltage sensor. Biophys $J$ 102, L44-46 (2012).

34. S. F. Altschul et al., Gapped BLAST and PSI-BLAST: a new generation of protein database search programs. Nucleic Acids Res 25, 3389-3402 (1997).

35. S. I. B. S. I. o. B. Members, The SIB Swiss Institute of Bioinformatics' resources: focus on curated databases. Nucleic Acids Res 44, D27-37 (2016).

36. H. M. Berman et al., The Protein Data Bank. Nucleic Acids Res 28, 235-242 (2000). 
37. S. Jo, J. B. Lim, J. B. Klauda, W. Im, CHARMM-GUI Membrane Builder for mixed bilayers and its application to yeast membranes. Biophys J 97, 50-58 (2009).

38. W. L. Jorgensen, J. Chandrasekhar, J. D. Madura, R. W. Impey, M. L. Klein, Comparison of simple potential functions for simulating liquid water. The Journal of Chemical Physics 79, 926-935 (1983).

39. A. P. Demchenko, S. O. Yesylevskyy, Nanoscopic description of biomembrane electrostatics: results of molecular dynamics simulations and fluorescence probing. Chem Phys Lipids 160, 63-84 (2009).

40. C. Kutzner, H. Grubmuller, B. L. de Groot, U. Zachariae, Computational electrophysiology: the molecular dynamics of ion channel permeation and selectivity in atomistic detail. Biophys J 101, 809-817 (2011).

41. M. Casciola, D. Bonhenry, M. Liberti, F. Apollonio, M. Tarek, A molecular dynamic study of cholesterol rich lipid membranes: comparison of electroporation protocols. Bioelectrochemistry 100, 11-17 (2014).

42. A. Polak et al., On the electroporation thresholds of lipid bilayers: molecular dynamics simulation investigations. J Membr Biol 246, 843-850 (2013).

43. D. Van Der Spoel et al., GROMACS: fast, flexible, and free. J Comput Chem 26, 17011718 (2005).

44. A. D. MacKerell et al., All-atom empirical potential for molecular modeling and dynamics studies of proteins. J Phys Chem B 102, 3586-3616 (1998).

45. A. D. Mackerell, Jr., M. Feig, C. L. Brooks, 3rd, Extending the treatment of backbone energetics in protein force fields: limitations of gas-phase quantum mechanics in reproducing protein conformational distributions in molecular dynamics simulations. $J$ Comput Chem 25, 1400-1415 (2004).

46. J. B. Klauda et al., Update of the CHARMM all-atom additive force field for lipids: validation on six lipid types. J Phys Chem B 114, 7830-7843 (2010).

47. H. J. C. Berendsen, J. P. M. Postma, W. F. Vangunsteren, A. Dinola, J. R. Haak, Molecular-Dynamics with Coupling to an External Bath. Journal of Chemical Physics 81, 3684-3690 (1984).

48. G. Bussi, D. Donadio, M. Parrinello, Canonical sampling through velocity rescaling. $J$ Chem Phys 126, 014101 (2007).

49. B. Hess, H. Bekker, H. J. C. Berendsen, J. G. E. M. Fraaije, LINCS: A linear constraint solver for molecular simulations. Journal of Computational Chemistry 18, 1463-1472 (1997).

50. U. Essmann et al., A smooth particle mesh Ewald method. The Journal of Chemical Physics 103, 8577-8593 (1995).

51. S. Jo, T. Kim, W. Im, Automated builder and database of protein/membrane complexes for molecular dynamics simulations. PLoS One 2, e880 (2007).

52. F. E. Regnier, W. Cho, in Proteomic and Metabolomic Approaches to Biomarker Discovery. (2013), pp. 197-224.

53. K. Vanommeslaeghe et al., CHARMM general force field: A force field for drug-like molecules compatible with the CHARMM all-atom additive biological force fields. $J$ Comput Chem 31, 671-690 (2010).

54. J. J. Irwin, T. Sterling, M. M. Mysinger, E. S. Bolstad, R. G. Coleman, ZINC: a free tool to discover chemistry for biology. J Chem Inf Model 52, 1757-1768 (2012).

55. O. Livnah, E. A. Bayer, M. Wilchek, J. L. Sussman, Three-dimensional structures of avidin and the avidin-biotin complex. Proc Natl Acad Sci U S A 90, 5076-5080 (1993). 
bioRxiv preprint doi: https://doi.org/10.1101/2019.12.28.889881; this version posted December 28, 2019. The copyright holder for this

preprint (which was not certified by peer review) is the author/funder. All rights reserved. No reuse allowed without permission. 
416 Figure legends

417 Figure 1. Molecular representations of Kv channel structures.

418 The crystal structures of A) Kv1.2, B) Kv1.2/Kv2.1and C) KvAP are shown embedded in a lipid 419 bilayer (Phosphorus atoms shown as orange spheres). The red arrows illustrate the different 420 translational motions that S4 is likely to undergo without exposing any hydrophobic residue 421 outside of the bilayer core. Such a translation seems not possible in the case of the KvAP crystal 422 structure, thus there is no arrow in panel C. The helices are colored as follows: S1: grey, S2: yellow, 423 S3: red, S4: blue. The arginine residues of the S4 N-ter are shown as sticks with carbon, nitrogen, 424 oxygen in black, blue and red.

Figure 2. The double bilayer system enables the explicit tuning of the membrane

\section{7 potential.}

Left) Molecular representation of two antiparallel bilayers with embedded VSDs. The proteins are represented and colored as in Figure 1 and the phosphorous atoms as well as the ions are depicted as spheres. Phosphorous, sodium and chloride are colored orange, violet and green. For clarity, water and lipid molecules have been removed. All the atoms shown constitute one simulated entity. Because of the periodic boundary conditions, the water and ions above and below each displayed bilayer communicate and form a single slab (EC: extracellular), which is isolated from the middle slab (IC: intracellular).

Right) The electrostatic potential along the bilayer normal (z) is shown adjacent to the molecular representation. We draw the potential at the beginning (0-20 ns, black) and at the end (last $20 \mathrm{~ns}$, red), which is normalized to $0 \mathrm{~V}$ at $\mathrm{z}=0$, i.e. in the E.C. The similarity of the two curves indicates that the charge imbalance manually set between the two compartments remained stable during the simulation. 
Figure 3. Salt bridge rupture upon polarization

A) S4 is straight and the Asp62-Arg133 salt bridge is intact in the X-ray structure. B)

Representative snapshot from a simulation conducted under polarized potential, taken after the charge transport, showing kinked S4 and broken salt bridge.

Figure 4. Co-occurrence of the salt bridge rupture and the kink formation in S4 upon polarization

A) Time series of the minimal distance between Asp62 and Arg133 (black, left y-axis) and the bending of S4 (blue, right y-axis). Mean \pm standard deviations from four pooled independent trajectories are shown. Before pooling, the time axis of each trajectory was set to 0 when the Asp62 - Arg133 distance goes above $3 \AA$ for the first time (red dashed vertical line). For comparison, the inset shows the S4 angle time evolution (Mean \pm standard deviation) of

trajectories conducted at Vm $=0 \mathrm{~V}(\mathrm{n}=62)$. B) The status of the Asp62-Arg133 salt bridge depends upon the membrane potential. Black lines, left y-axis: distance between Asp62 and Arg133 as a function of simulation time. Red lines, right y-axis: membrane potential as a function of simulation time. At $\mathrm{t} \sim 90 \mathrm{~ns}$, rupture of the salt bridge. At $\mathrm{t}=200, \mathrm{Vm}$ manual sign inversion by ion displacement (red arrow). At t 210ns, restoration of the salt bridge.

\section{Figure 5. The kink in $\mathrm{S} 4$ allows binding of avidin to biotinylated residues in the}

middle of S4. Snapshots extracted from steered molecular dynamics simulations initiated with a VSD in which the S4 helix is straight (A: $t=50 \mathrm{~ns}$ ) or kinked (B: $t=0 \mathrm{~ns}, \mathrm{C}: \mathrm{t}=36 \mathrm{~ns}$ ). Avidin is colored in green, and biotin is shown in stick representation with carbon black, oxygen red and hydrogen grey. The distance between biotin and the $\mathrm{C} \alpha$ (black sphere) of ILE127 is highlighted. D) Time series of the distance between the carboxylic group of biotin 
468 harboring the kink in S4 (red). For control, the distance between the biotin and its binding

469 site to avidin is shown (right y axis). Pulling forces were applied between the biotin and the

470 ILE127 $\mathrm{C} \alpha$, and between the biotin and the avidin binding site (see Methods).

471

472 Table 1. Summary of the charge transport events observed during this study. 


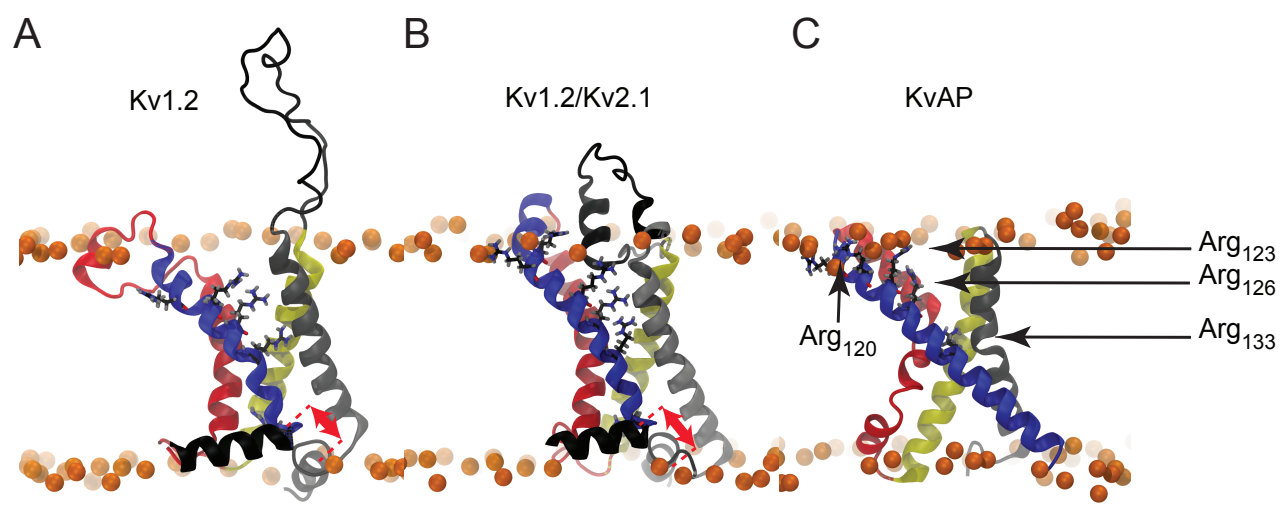

Figure 1
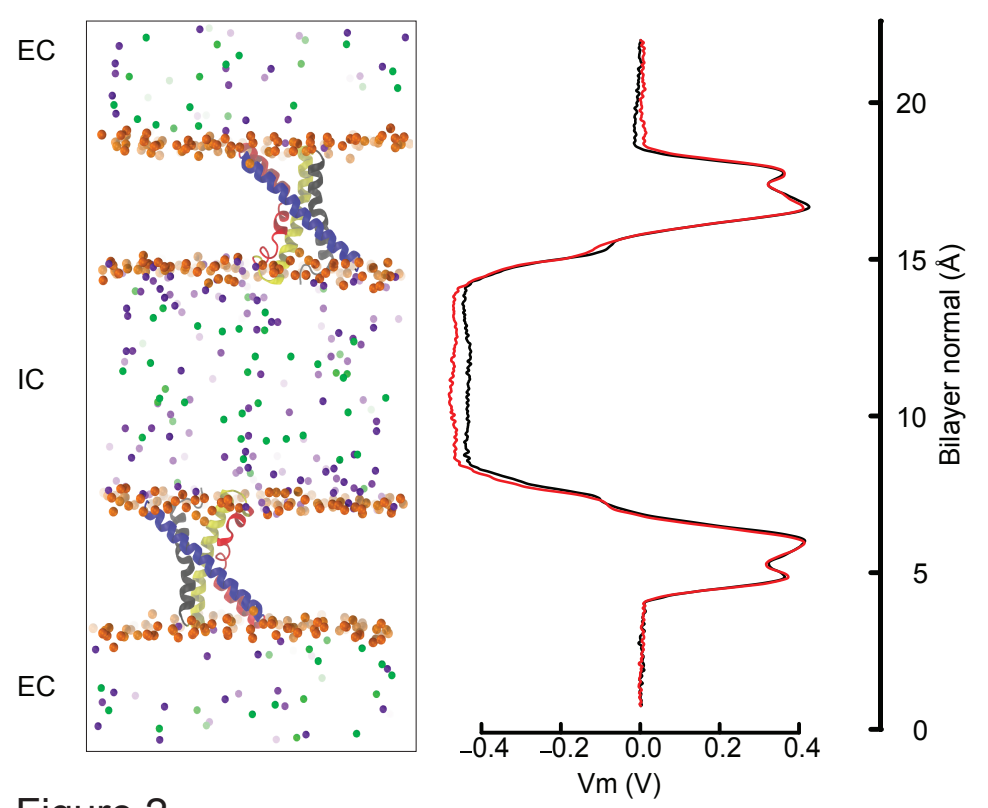

Figure 2
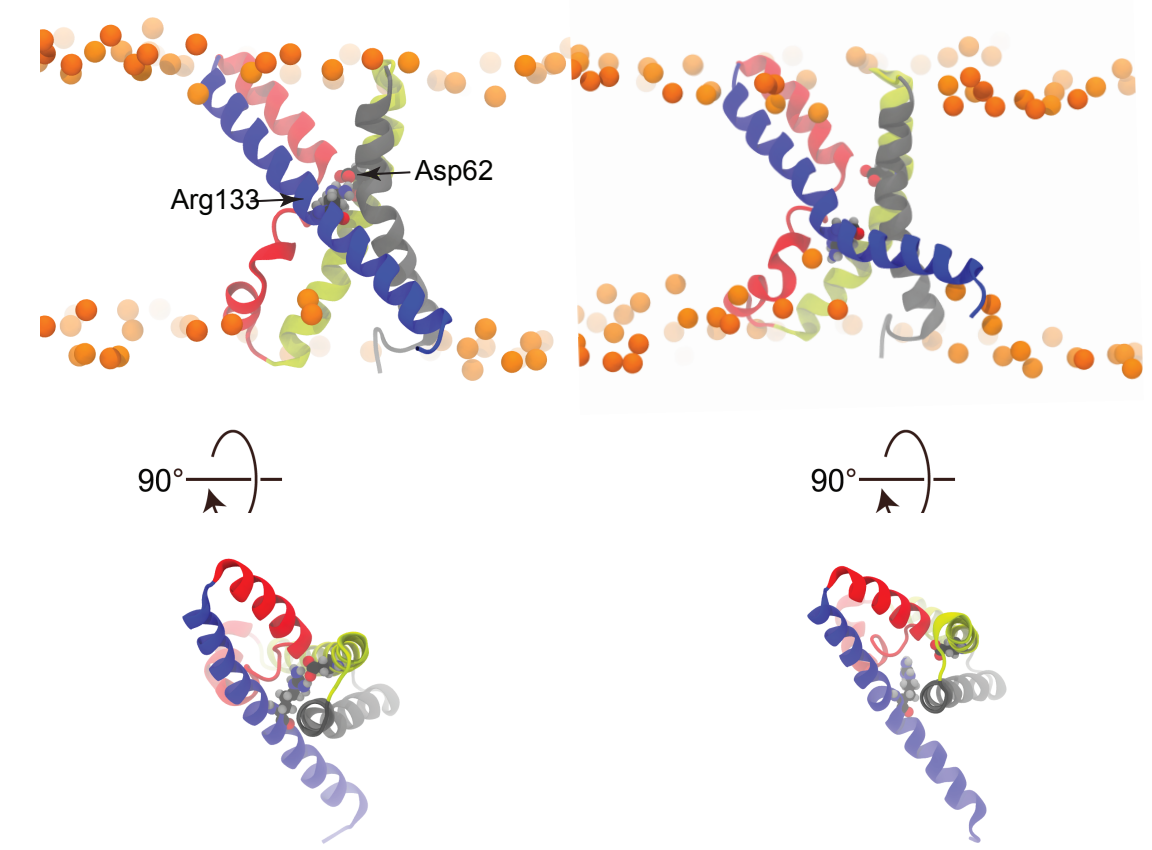

Figure 3 
A
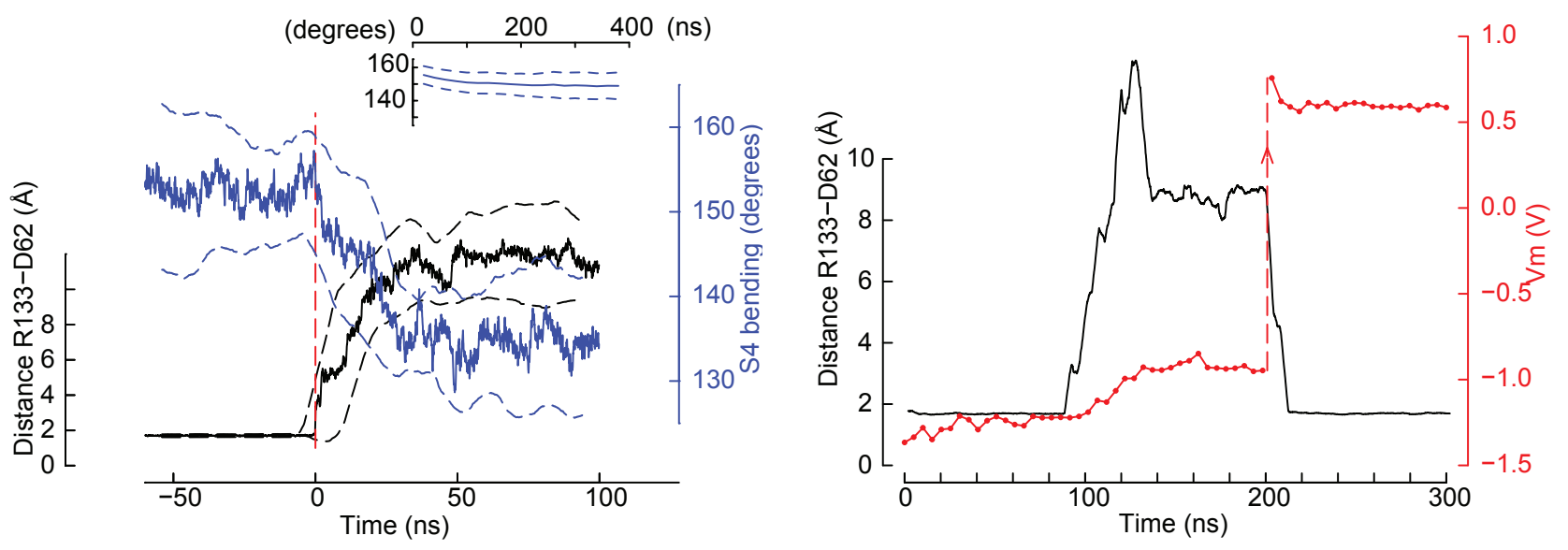

Figure 4

A

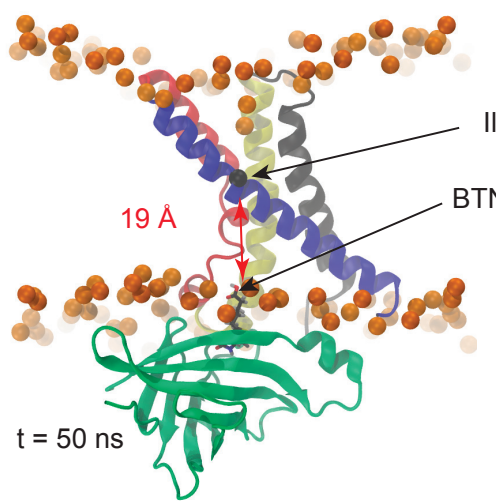

D

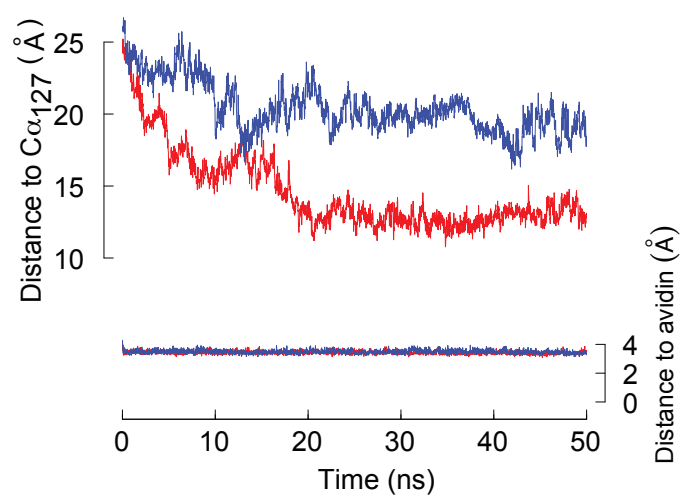

Figure 5

\section{B}

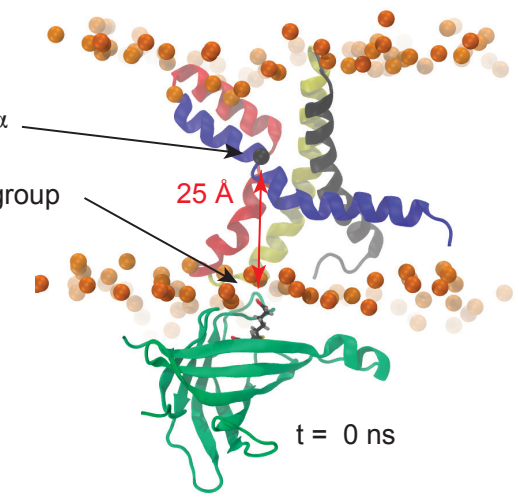

C

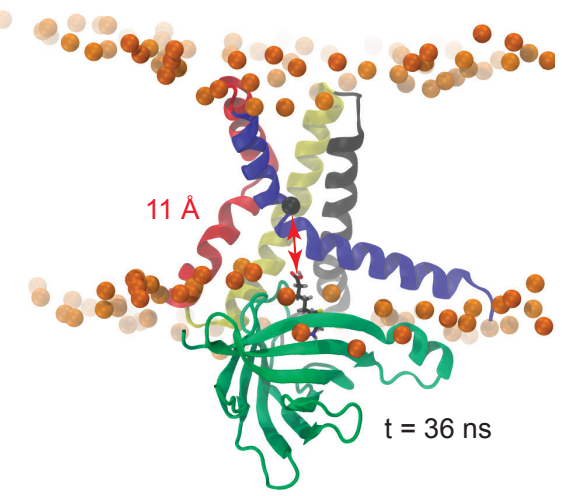




\begin{tabular}{|l|c|c|}
\cline { 2 - 3 } \multicolumn{1}{c|}{} & Polarized & Depolarized \\
\hline Charge transport & 4 & 0 \\
\hline No charge transport & 8 & 53 \\
\hline
\end{tabular}

\section{Table 1}




\section{Supplementary Material}

\section{The voltage-sensing mechanism of the KvAP channel involves breaking of the $S 4$ helix}

Olivier Bignucolo1,2,3,\#, Simon Bernèche1,2

1SIB Swiss Institute of Bioinformatics 2Biozentrum, University of Basel, Switzerland ${ }_{3}$ Current address: Faculty of Biology and Medicine, University of Lausanne, Switzerland \#Correspondence: olivier.bignucolo@unil.ch

\section{Sequence analysis suggests a potential target for antibiotics research}

The specific features of the KvAP S4 N-ter sequence is not seen in any eukaryotic voltagegated ion channel. The Gly residue at which the observed kink occurs is located 8 positions downstream from the S4 basic residues. As shown in Supplementary Table 1, the most resembling eukaryotic sequences contained a much shorter segment, generally 3 residues, connecting the last S4 basic residue and the next Gly. However, we found that several prokaryotic potassium voltage-gated channels displayed a similar sequence as the KvAP S4 Nter. Many of them belong to the anaerobic Bacteroides species, which are of significant clinical relevance. Bacteroides fragilis infections display a mortality of $20 \%$, which rises to more than $60 \%$ if left untreated (1). A study on bacteremia, reveals that while the incidence of anaerobic bacteremia is relatively low (less than $3 \%$ ), the associated mortality is higher than $20 \%$ (2). In the same line, Bacteroides pyogenes causes serious human wound infections $(3,4)$ whereas Bacteroides thetaitoamicron, which can exacerbate Escherichia coli (E.coli) and Clostridium difficile infections, is the second most common infectious anaerobic gram-negative bacteria(5). Thus, the prokaryotic specific mechanism identified in the current study may be 
used as a selective target addressing several lethal pathogens, some of them being a cause for major concern in the context of increasing reported cases of antimicrobial ( $\beta$-lactam, Carbapenems and other antibiotics) resistance (6-9). Mutation experiments involving the $E$. Coli Kch potassium channel suggested that it maintains the membrane potential and could prove essential under certain stress conditions, like higher external potassium concentration(10). Further, in support to the pertinence of potassium channels as potential antibacterial targets, gastric colonization by Helicobacter pylori lacking its potassium channel HpKchA is impaired (11).

\section{Ion and water transport}

In two instances, we observed a potassium ion moving from the extracellular to the intracellular compartment, as well as several water molecules. In a previous molecular dynamics study, Freites et al. reported the formation of a water pore through the KvAP VSD, pointing the Asp62Arg133 salt bridge rupture as a requirement for water transport (14). However, we propose that this opening is rather transient, and that a further sliding of S4, though not observed in our rather short simulations, would allow Arg133 to form new interactions with acidic residues located more toward the intracellular side, namely Asp72 in S2 and Glu93 in S3. These new interactions would then restrict the transient opening through the VSD.

\section{The pore domain and the membrane}

In this study, only the voltage sensor of KvAP is included in the simulation systems, without the pore domain. The conformation and dynamics of the isolated VSD is expected to be different from that observed in presence of the pore domain. Nevertheless, the polarization is the driving force inducing the disruption of the salt bridge and the displacement of the positively charged upper segment of S4. The direction and magnitude of this force does not depend on the fact that 
the VSD is linked or not to the pore domain, and thus these two events are expected to happen in the case of a whole channel as well. In our simulations, the intracellular segment of S4 moved unconstrained, with a tendency to orient parallel to the membrane. In principle, this movement could be different in the case of the whole channel. The comparison of the whole chain structure and the structure of the voltage sensor alone shows, however, that the conformation of the S1S4 helices in the open state is not affected by the pore domain (Suppl. Figure 2).

We noticed a narrowing of the lipid bilayer in the vicinity of the protein. Even residues Arg 123 and Arg126, which are located relatively deep towards the middle of the bilayer, formed hydrogen bonds with the lipids. The water filled cavities, on both sides of the membrane, also induced rearrangements of the phospholipids. As shown in Figure 3B, a few phosphate groups entered in the water filled cavity, although they generally remained at the most external part of it. We wondered whether the formation of the kink, which may further enlarge the water filled cavity on the intracellular side, would affect the structure of the bilayer. As shown in Supplementary Figure 1, the intracellular leaflet thickness was $\sim 20 \AA$ resp. $17 \AA$ for lipids situated far from resp. near the VSD. However, the thinning of the bilayer was the same whether S4 formed a kink or not. To be more precise, we further plotted the thickness of the membrane as a function of the bending of S4. We also analyzed the thickness of the lower leaflet as a function of the height of the last residue of S4, defined as the distance along the normal to the bilayer between the C $\alpha$ atom of Leu148 and the average height of the C316 atoms of the lipids, representing the center of the bilayer. In both analyzes (data not shown), there was no relationship between the thickness of the leaflet and the shape of S4, which demonstrates that the kink did not affect the penetration of the lipid head groups into the membrane. On the other hand, the polar head groups tended to reorient in the vicinity of a transmembrane protein. We then wondered whether the formation of the kink in S4 would modify the polar head orientation. The orientation of the phosphocholine head groups is defined by the angle between 
the Phosphorus to Nitrogen vector and the normal to the bilayer. The average value of this angle was $\sim 71$ degrees resp. $\sim 64$ for lipids situated far from resp. near the VSD. The kink in S4 has no impact on the reorientation of the lipid polar heads. It is thus concluded that whereas the membrane structure was modified in the vicinity of the channel, these rearrangements were not affected by the disruption of the Asp62-Arg133 salt bridge and the kink of S4. 
bioRxiv preprint doi: https://doi.org/10.1101/2019.12.28.889881; this version posted December 28, 2019. The copyright holder for this preprint (which was not certified by peer review) is the author/funder. All rights reserved. No reuse allowed without permission.

A)

Lipids $<7 A$ from VSD

Lipids > 30A from VSD
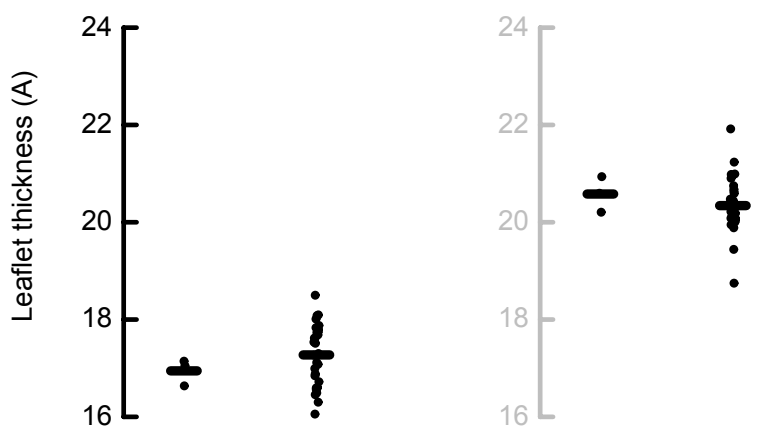

S4 kinked S4 straight

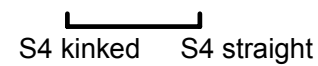

B)
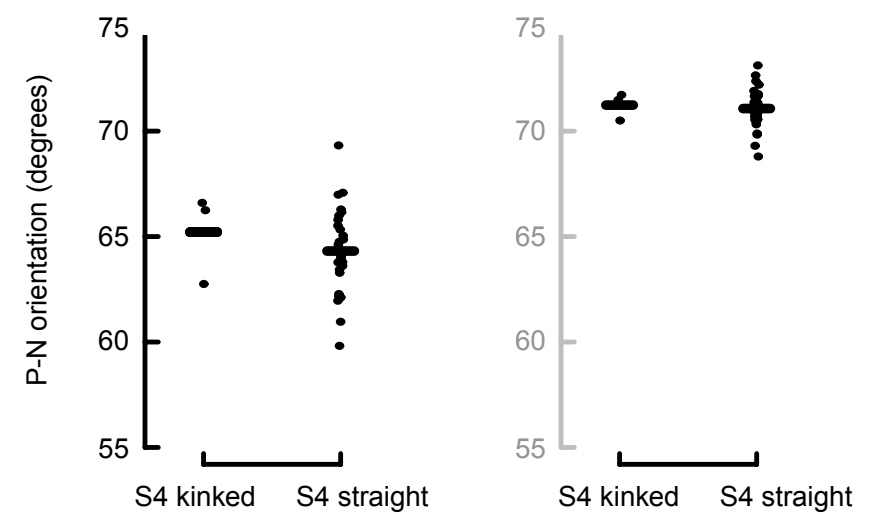

Supplementary figure 1

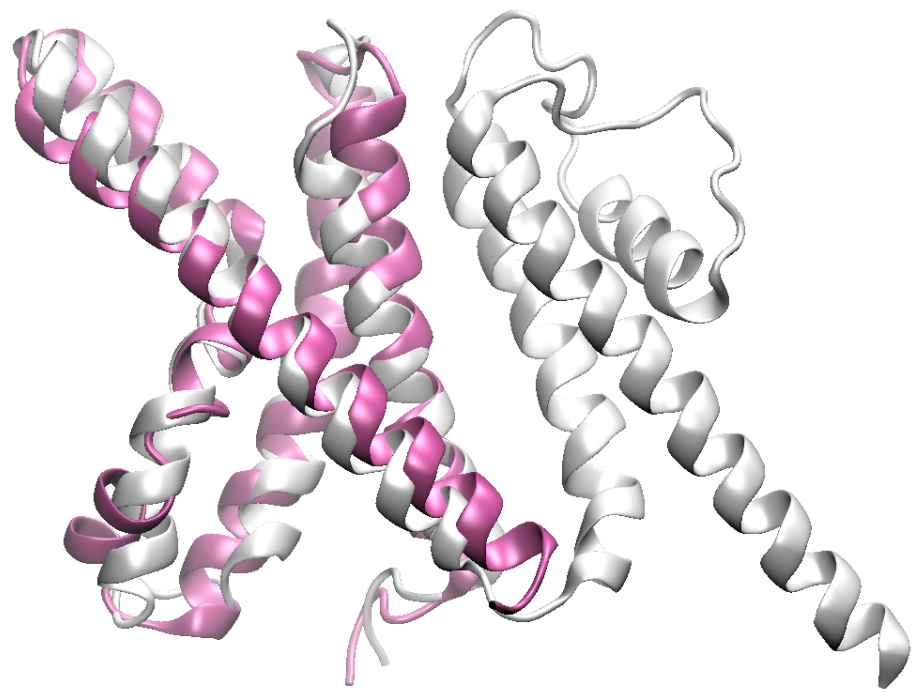

Supplementary figure 2 
bioRxiv preprint doi: https://doi.org/10.1101/2019.12.28.889881; this version posted December 28, 2019. The copyright holder for this

preprint (which was not certified by peer review) is the author/funder. All rights reserved. No reuse allowed without permission.

\section{A. Prokaryotes}

Aeropyrum pernix Bacteroides pyogenes Bacteroides thetaiotamicron Halomonas pantelleriensis Bacillus wakoensis Bacteroides faecichinchillae Pontibacter virosus Bacteroides ovatus Planococcus donghaensis Bacteroides finegoldii Pedobacter cryoconitis Flavobacteriales bacterium Bacteroides plebeius Bacteroides fragilis Prevotella disiens Bacteroides caccae Porphyromonas gingivalis consensus

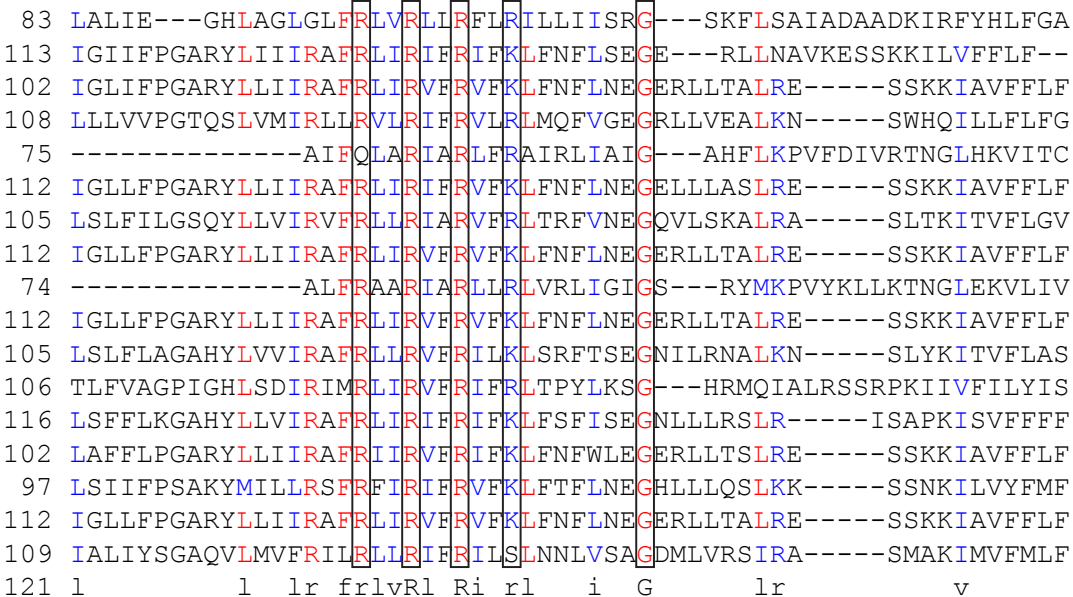

\section{B. Eukaryotes}

KvAP Aeropyrum pernix Shaker Stylophora pistillata Shaker Folsomia candida Shaker Nothobranchius furzeri KV subf. A Crassostrea gigas Kv subf. A Mizuhopecten y. Kv subf. A Gasterosteus a. Kv subf. A Hydra vulgaris Kv subf. A Salmo salar Kv subf. A Ciona intestinalis $\mathrm{Kv}$ subf. A Mus musculus Kv subf. A Ictalurus p. Kv subf. A Rattus norgegicus $\mathrm{KV}$ subf. D Clonorchis s. Kv subf. D Shistosoma j. Kv Loa loa Kv Hypsibius dujardini Kvs 5 Pristionchus pacificus Kv Nematostella vectensis Kv Harpegnathos saltator Cation channel Tetrahymena $t$. Transporter Teladorsagia c. Kv Nematostella vectensis Kvs2 Caenorhabditis elegans Kv1.2 homo sapiens

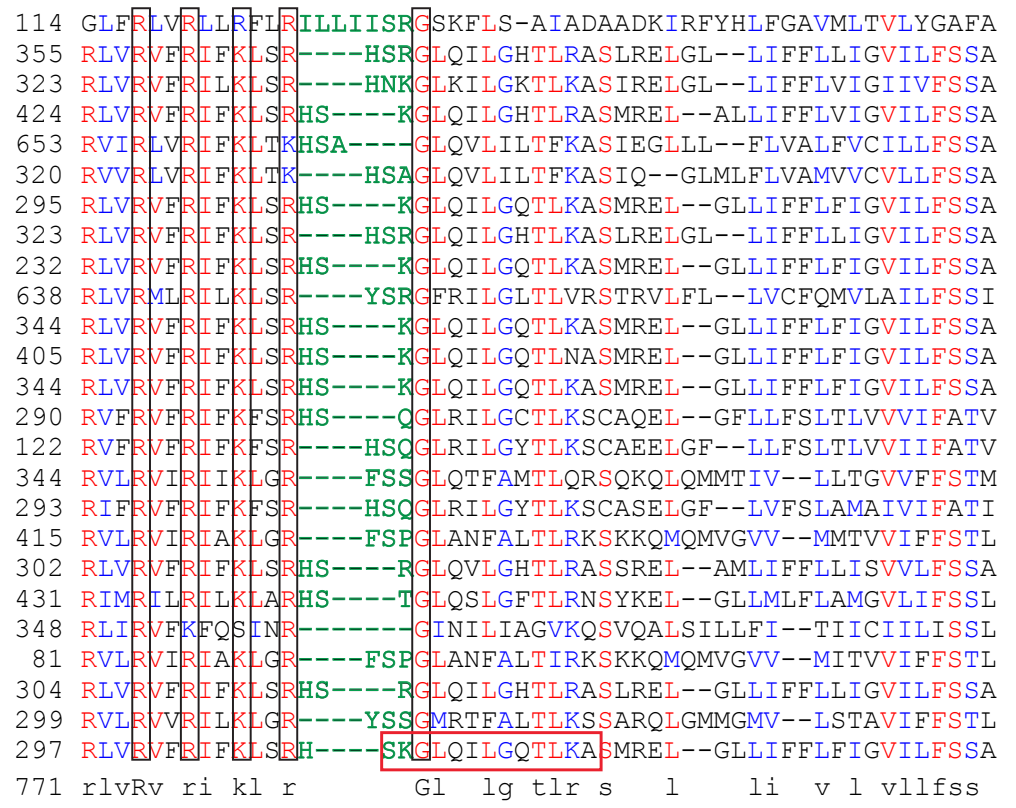

\section{Supplementary Table 1}

